\title{
On electromagnetic precursors to the Hokkaido earthquake in September, 2018 and consideration of lithosphere-atmosphere-ionosphere coupling
}

\author{
Masashi Hayakawa ${ }^{1,2,3}$, Alexander Rozhnoi ${ }^{4}$, Maria Solovieva ${ }^{4}$, and Shih-Sian Yang ${ }^{5,1}$ \\ ${ }^{1}$ Hayakawa Institute of Seismo Electromagnetics, Co. Ltd. (Hi-SEM), UEC (University of Electro- \\ Communications) Alliance Center \#521, 1-1-1 Kojima-cho, Chofu, Tokyo 182-0026, Japan \\ ${ }^{2}$ UEC, Advanced Wireless \& Communications Research Center, 1-5-1 Chofugaoka, Chofu Tokyo, 182-8585, Japan \\ ${ }^{3}$ Geoscent Technologies Inc., 2-8-11 Akasaka, Minato-ku, Tokyo, 107-0052, Japan \\ ${ }^{4}$ Institute of Physics of the Earth, Russian Academy of Sciences, 10 B Gruzinskaya, Moscow 123995, Russia \\ ${ }^{5}$ Independent researcher, Jhongli P.O. Box 9-11, Taouyuan 32099, Taiwan
}

\begin{abstract}
Electromagnetic precursors have been investigated for a recent relatively large earthquake (EQ) with magnitude $M=6.7$ occurred in the Hokkaido Island, Japan on 5 September (UT), 2018 on the basis of coordinated data on the lower ionospheric perturbation with subionospheric VLF/LF propagation anomalies monitored in Japan and in Russia, on the upper F region anomaly with GIM (global ionosphere map) TEC (Total Electron Content) and on the stratospheric behavior with the use of ERA5 temperature profile data. It is found that there were observed the clearest VLF/LF propagation anomalies indicative of seismogenic lower ionospheric perturbations above the EQ epicenter on 4 and 5 September before the EQ and 6 September even after it because this period was geomagnetically quiet. As for the F region, an anomaly in TEC over the EQ epicenter appeared only on 5 September, but it is quite uncertain whether this anomaly is related to either a pre-EQ effect or a geomagnetic storm effect. Then, we have tried to find if there were some seismo-related AGW (atmospheric gravity wave) activities in the stratosphere, but though we have found a lot of anomalies, they are highly likely to be associated with the activities by convective weather systems. Even though we have recently identified the first convincing direct evidence on the Atmospheric oscillation hypothesis in the lithosphere-atmosphere-ionosphere coupling (LAIC) mechanism for the 2016 Kumamoto EQ [Yang et al., 2019], this paper aimed initially to obtain further evidence on this channel. However, the above experimental findings may provide a negative result in this direction; that is, this Atmospheric oscillation channel is unlikely to be in operation for the present 2018 Hokkaido EQ, suggesting other channels in the LAIC effect. So we have made extensive discussions on this LAIC mechanism for this Hokkaido EQ with special reference to the 2016 Kumamoto EQ and the 2011 Tohoku EQ.
\end{abstract}




\section{Introduction}

It is recently agreed that electromagnetic phenomena do take place prior to earthquakes (EQs) [Hayakawa and Molchanov 2002; Pulinets and Boyarchuk, 2004; Molchanov and Hayakawa 2008; Hayakawa, 2015; Sorokin et al., 2015; Ouzounov et al., 2018]. Especially, the upper atmosphere or the ionosphere is known to be very sensitive to pre-EQ lithospheric activity: that is, perturbations not only in the lower ionosphere (as seen by subionospheric VLF/LF propagation) [Rozhnoi et al., 2004; Hayakawa, 2011; Rozhnoi et al., 2013; Hayakawa et al 2018], but also in the upper F region as detected by ionosondes, TEC observation, etc. [Hayakawa and Molchanov 2002; Pulinets and Boyarchuk, 2004; Molchanov and Hayakawa 2008; Hayakawa, 2015; Sorokin et al., 2015; Ouzounov et al., 2018; Liu et al., 2018] are known to occur a few days to about one week before major EQs. The generation of those seismo-ionospheric perturbations is being envisaged in the context of lithosphere-atmosphere-ionosphere coupling (LAIC), which is the main goal of our EQ predictology, but is poorly understood at the moment.

There have already been proposed a few hypotheses for this LAIC. The first is "Chemical (and associated electric field)" channel [Pulinets et al 2018], in which radon emanation is the main player, leading to the change in atmospheric electric field and then to the ionospheric perturbation. The similar "Electrostatic" channel has been proposed, in which stress-activated positive holes are generated on the Earth's surface, leading to the generation of a large electric field [Freund, 2009]. Whatever the agent of electric field generation either by radon or by positive holes, these two hypotheses are just through electric fields. The third is completely different from those channels, and is "Atmospheric oscillation (Acoustic wave (AW) / atmospheric gravity wave (AGW))" hypothesis [Pulinets and Boyarchuk, 2004; Lizunov and Hayakawa, 2004; Hayakawa et al., 2011; Yang et al., 2019] (often called simply AGW channel), in which water, radon, gas emanation etc. before an EQ could excite the AGW oscillations and those AGWs propagate upwards and obliquely and result in the lower ionospheric perturbations [Hayakawa et al., 2018; Lizunov and Hayakawa, 2004].

There have been accumulated a lot of indirect evidence in support for the Atmospheric oscillation hypothesis in the LAIC [Hayakawa, 2011; Rozhnoi et al., 2013; Lizunov and Hayakawa, 2004], and a summary of those evidence is given in Lizunov and Hayakawa [2004]. However, there has always been a serious criticism on this hypothesis that there have been no reports on any associated changes at the nearthe-ground surface or in the ground of exciting such AGWs. Recently a paper has been published on the 
direct evidence of generation and propagation of seismo-AGWs in the stratosphere (altitude $=15-50 \mathrm{~km}$ ) by using the reanalysis temperature profile data for a particular 2016 Kumamoto EQ [Yang et al., 2019]. This study has lent definite evidence on the Atmospheric oscillation hypothesis in the LAIC mechanism. A further study has been carried out for the 2011 Tohoku EQ and has been submitted elsewhere again in favor of the Atmospheric oscillation channel [Yang et al, 2019].

In order to elucidate the mechanism of LAIC, it is highly required for us to deal with more case studies as done in [Yang et al., 2019] with utilizing numerous observational parameters in different regions, i.e. in the ionosphere (lower part and upper F region), in the stratosphere, and preferably in the lithosphere.

This paper is aimed to study a recent large EQ in Hokkaido with magnitude of $M=6.7$, with the use of VLF propagation data observed in Japan and in Russia for the study of lower ionosphere, GIM TEC data for the study of upper ionosphere, and reanalysis temperature profile data for the stratosphere, and finally we wanted to obtain further evidence on the Atmospheric oscillation hypothesis in the LAIC for this Hokkaido EQ. Nevertheless we have obtained intuitively the results in opposition to this direction, and so we will have extensive discussion on the LAIC effect for this Hokkaido EQ with special reference to our recent findings for the 2016 Kumamoto EQ and the 2011 Tohoku EQ

\section{EQ treated in this paper}

A rather big EQ happened in the Hokkaido Island at the geographic coordinates $\left(42.69^{\circ} \mathrm{N}, 142.01^{\circ} \mathrm{E}\right)$ on 6 September, 2018 at $3 \mathrm{~h} \mathrm{7m} \mathrm{59s} \mathrm{JST} \mathrm{(or} 5$ September, $18 \mathrm{~h} 7 \mathrm{~m} \mathrm{59s}$ UT). Its magnitude was $\mathrm{M}=6.7$ and its depth was $37 \mathrm{~km}$. Despite this magnitude, the Japanese seismicity intensity reached the highest level of 7 , which has never been recorded in Hokkaido before.

\section{Possible EM precursors to this EQ}

How about the precursors for the 2011 Tohoku mega-EQ? There were in fact precursors, although most of them were only recognized afterward. There are also encouraging signs for future developments in short-term prediction. This part is based on Hayakawa [2018], mainly referring to the results by Japanese colleagues. However, we have also cited the corresponding some precursors by foreign scientists with satellite observations (some of them are shown in the book by Ouzounov et al. (Eds) [2018]. 


\subsection{Perturbations in the lower ionosphere as seen by subionospheric VLF/LF propagation data}

We have been monitoring the lower ionospheric perturbations [e.g. Molchanov and Hayakawa, 2008; Hayakawa, 2015; Hayakawa et al., 2018] with the use of our own Japanese VLF/LF network composed of about $10 \mathrm{VLF} / \mathrm{LF}$ receiving stations, each of which is equipped with the same receiving system consisting of VLF and GPS antennas, pre-amplifier, VLF receiver, sound card and signal processing algorithm. The details of the equipment are given in [Hayakawa, 2011; Hayakawa et al., 2018]. At each station we detect simultaneously subionospheric signals from two Japanese VLF/LF transmitters (JJY (Fukushima, $\mathrm{f}=40 \mathrm{kHz}$ ) and JJI (Miyazaki, f=22.2 kHz)) and three foreign transmitters (NLK (Jim Creek, USA), NPM (Hawaii) and NWC (west Australia)). Among many observation stations, we have chosen one particular station of Wakkanai (abbreviated as WKN) (geographic coordinates: $45.52^{\circ} \mathrm{N}, 141.95^{\circ} \mathrm{E}$ ) suitable for the present study.

Our Russian colleagues have also been observing VLF/LF transmitter signals at PetropavlovskKamchatka (PTK) $\left(53.09^{\circ} \mathrm{N}, 158.55^{\circ} \mathrm{E}\right)$ and Yuzhno-Sakhalinsk (YHS) $\left(46.95^{\circ} \mathrm{N}, 142.75^{\circ} \mathrm{E}\right)$, and the system of this VLF/LF reception is very similar to Japanese.

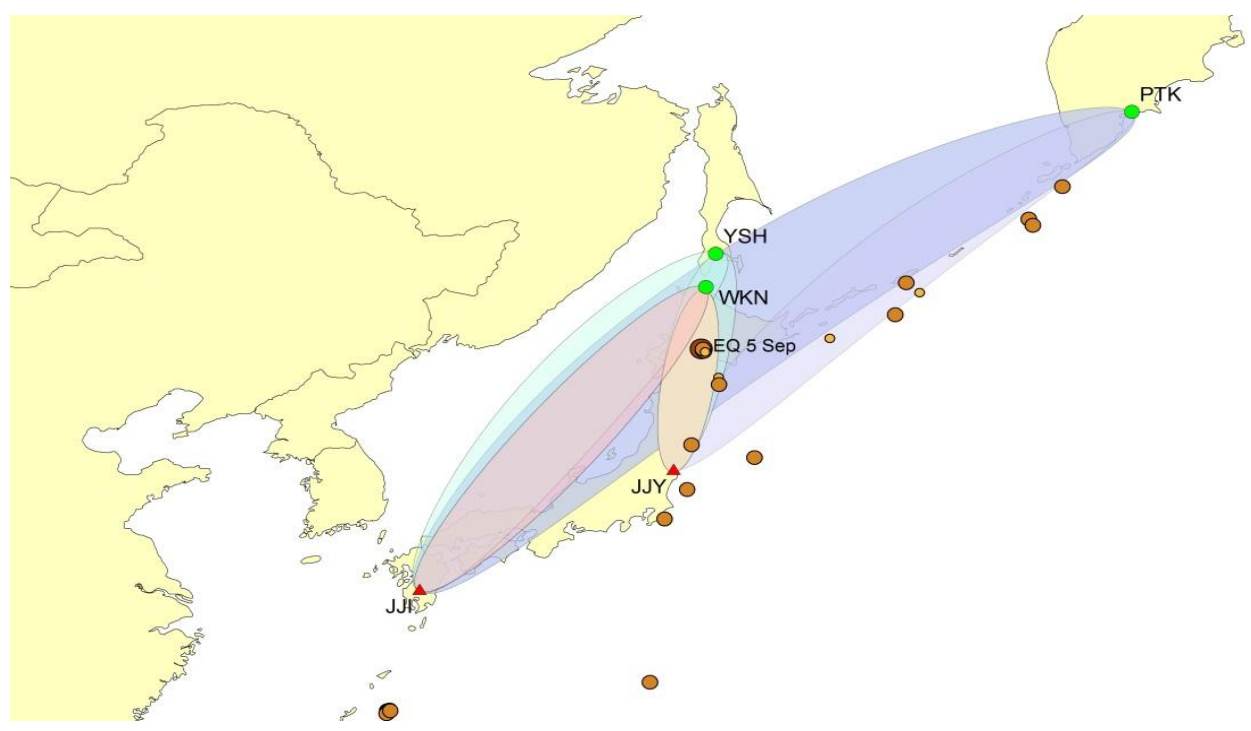

Figure. 1 A map of two Japanese VLF/LF transmitters (JJY (in Fukushima) and JJI (Miyazaki) (in red triangles) and VLF/LF observing stations (WKN (Wakkanai) in Japan and Petropavlosk-Kamchatka (PTK) and YuzhnoSakhalinsk (YHS) in Russia (all indicated by green circles)). Fresnel zones for JJY-WKN, JJI-WKN, JJY-PTK, and JJI-PTK are indicated by the corresponding ellipses. EQs with magnitude greater than 5 occurred in August and September, 2018 are plotted in brown circles as well, with the size proportional to EQ magnitude. The target Hokkaido EQ is shown by EQ 5 Sep. 
Figure 1 illustrates the relative location of two Japanese VLF/LF transmitters (JJY and JJI) indicated by red triangles, and our relevant receiving stations of WKN, PTK and YHS (indicated by green circles), together with EQs occurring in the Japanese and Russian areas in August and September, 2018 with magnitude greater than 5 (indicated by brown circles). The size of each circle is proportional to EQ magnitude, and our target EQ (Hokkaido EQ) is indicated by the signature of EQ of 5 September in the figure. The Fresnel zones of different paths are plotted as ellipses. Unfortunately the observation situation at YHS was not so good in both months, so that the corresponding Fresnel zones of JJY-YHS and JJI-YHS were not plotted in the figure.
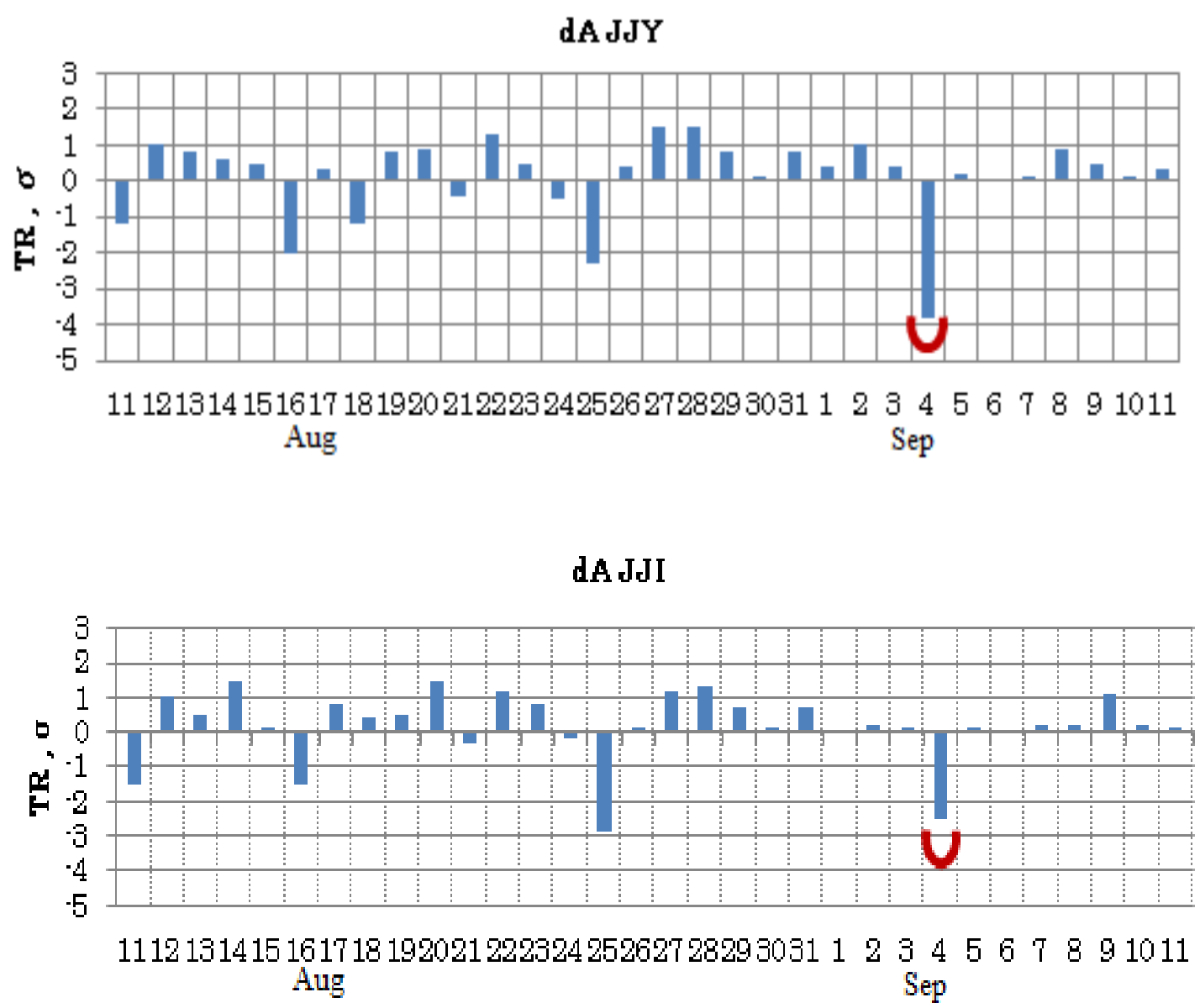

Figure. 2 Propagation characteristics observed at WKN during one month around the EQ date. The upper panel refers to JJY, while the bottom, to JJI. Both panels are the nighttime average amplitude with respect to the average during the previous one month $(\mathrm{dA})$, normalized with its standard deviation $(\sigma)$ during preceding one month (socalled Trend) (in blue). An anomaly marked is characterized by the depletion in trend exceeding $-2 \sigma$. 
Figure 2 illustrates the VLF/LF propagation characteristics observed at WKN for the two propagation paths of JJY-WKN (upper panel) and JJI-WKN (lower panel). Both panels refer to the results of "Trend", which is defined by the average nighttime amplitude relative to the mean value (during preceding one month) normalized by its standard deviation $(\sigma)$ during the preceding one month. The period of presentation is one month around the EQ date. Hayakawa et al., [2011, 2018] have demonstrated that a VLF/LF anomaly is characterized by the depletion in amplitude such as depletion exceeding $-2 \sigma$. The upper panel for the JJY-WKN path has exhibited the very significant depletion in amplitude exceeding $3 \sigma(-3.6 \sigma)$ on 4 September (in UT), just 1 day before the EQ. On the same day there was observed the corresponding anomaly on another path of JJI-WKN, but the depletion is not so much, just exceeding $-2 \sigma$. WKN is located at the highest latitude in Japan, so that we are afraid that VLF data there might be influenced by geomagnetic activity. So we have plotted in the top panel of Figure 3 the temporal evolution of Dst as an index of geomagnetic activity in August and September, and we have found that these LF propagation anomalies are occurring during a period of low geomagnetic activity and so they are likely to be seismogenic. And the difference in the value of amplitude depletion can be satisfactorily explained in terms of the relative position of each propagation path to the EQ epicenter, as seen from Figure 1. Another VLF anomaly is clearly seen on August 25, but this is apparent to be the effect of a geomagnetic storm on the same day. Figure 1 shows that the EQ epicenter was located well inside the Fresnel zone of JJY-WKN, and so we could expect a remarkable anomaly. On the other hand, the perturbed region or EQ preparation zone for this EQ is big enough [Dobrovsky et al., 1979] to overlap the propagation path of JJI-WKN, leading to the VLF anomaly, but less obvious. We will try to infer the scale of the perturbation later. Now we move on to the Russian VLF/LF results. Only the nighttime amplitude is presented in each hour in Figure 3, nearly the same as in Figure 2 (but Figure 2 refers to the average value of nighttime amplitude). Bottom two panels of Figure 3 illustrate the temporal variation of nighttime amplitude, $\mathrm{dA}(\mathrm{t})$. The upper refers to JJY-PTK and the lower, to JJI-PTK. The horizontal dotted lines in both figures indicate the lines of $-2 \sigma$. For our comparison the top panel of Figure 3 indicates the geomagnetic activity expressed by Dst (in nT), and the second refers to the occurrence (and magnitude) of EQs in Figure 1. The period of early August till the end of August was geomagnetically quiet, but the late August to September was very disturbed geomagnetically because there occured a few geomagnetic storms. We can record on the path of JJY-PTK a clear anomaly exceeding $-2 \sigma$ on the days of 4, 5 and 6 September, just around the EQ date, but mainly before the EQ. So, a combination of these 
Russian VLF/LF results with the previous Japanese results in Figure 2 enables us to regard that the VLF/LF propagation anomalies on these days are likely to be a precursor to this EQ.

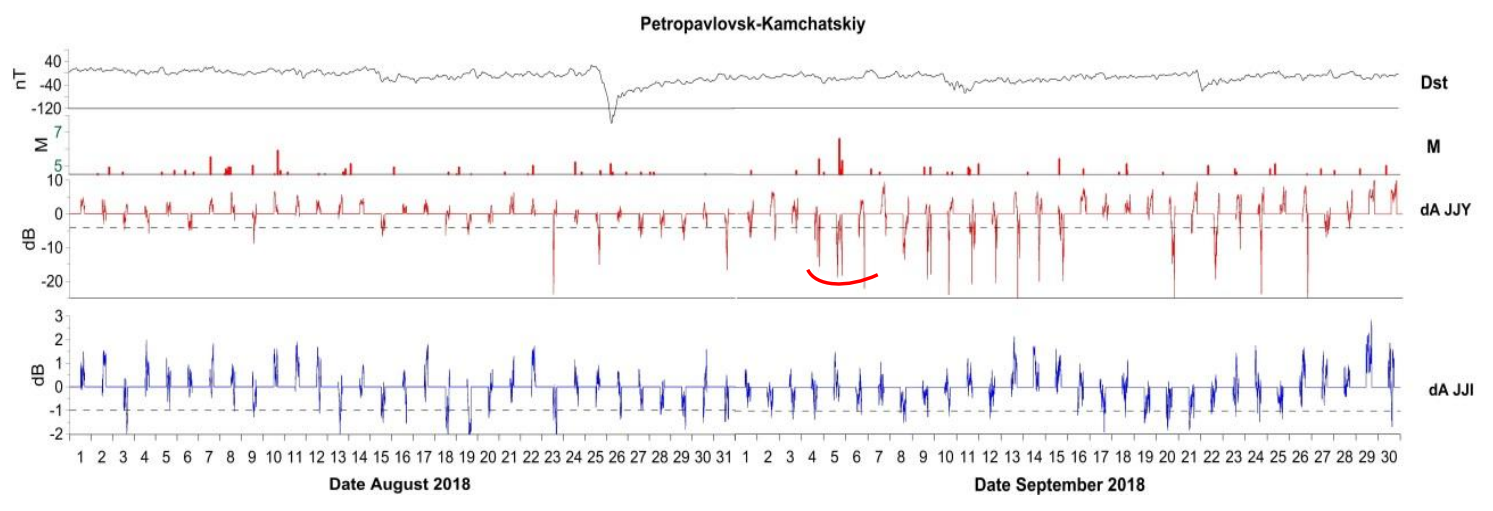

Figure. 3 Propagation characteristics observed at PTK. The top panel indicates the temporal evolution of geomagnetic activity expressed by Dst, and the second panel illustrates the evolution of seismic activity, occurrence of EQs (in magnitude). Similarly to Figure 2 the third and fourth panels illustrate the differential nighttime amplitude dA for the JJY and JJI signals, respectively. The horizontal broken lines are $-2 \sigma$ lines as the threshold of anomaly, and a VLF anomaly is marked on the path of JJY-PTK. Unlike Figure 2, the diurnal variation at night $(\mathrm{dA}(\mathrm{t}))$ is plotted.

Long-lasting and stronger (decrease in amplitude exceeding $-2 \sigma$ ) anomalies are observed at PTK as in Figure 3 in September (10-16 and 20-26 September). Because the propagation path for PTK is located at mid-latitudes, then those anomalies can be caused either by geomagnetic activity or with a generally increased seismic activity as seen from Figure 3 [Rozhnoi et al., 2004].

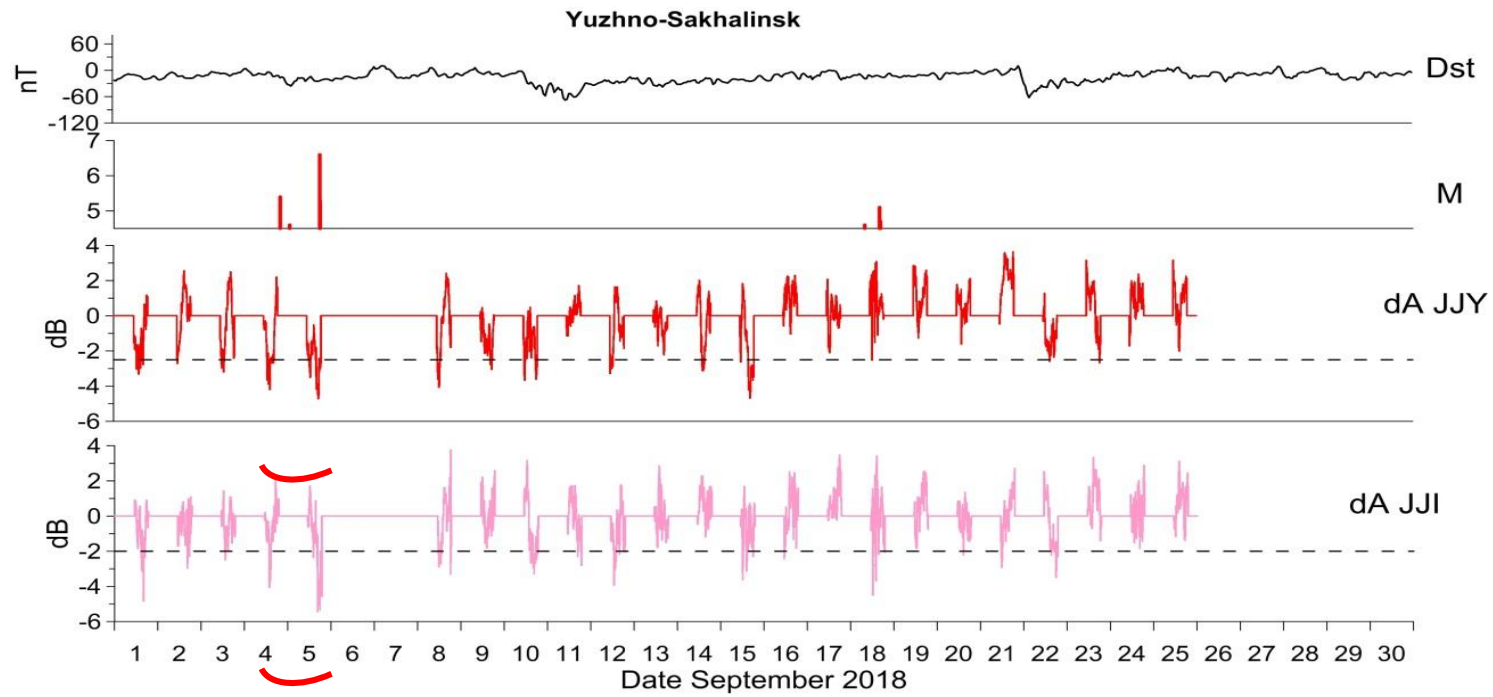

Figure 4: The same as Figure 3, but at the station of YHS. Anomaly parts are also marked. 
As mentioned before, the data at YHS are not so good because of discontinuities in the observation due to the failure of power supply, but we dare to present the VLF/LF data there. Figure 4 is the result, and the third panel refers to JJY, while the bottom, to JJI. In consistence with the result at WKN and considering the closeness between YHS and WKN, we could identify the corresponding anomalies on 4 and 5 September before the EQ. This Figure will provide us with a further support to the presence of VLF/LF propagation anomalies on the relevant days as a signature of seismo-lower ionospheric perturbation.

Here we attempt to estimate roughly the possible size of the lower ionospheric perturbation with making full use of the information from our VLF/LF network observation. In addition to the data from one particular station of WKN as presented in this paper, we have looked at the data at two more stations close to the EQ epicenter; one is Nemuro in Hokkaido and the other is Kisakata (Akita), and also we have examined the propagation characteristics for the foreign transmitters (NLK and NWC (The transmitter of NPM was under maintenance)). A whole combination of consideration of those propagation characteristics we can infer the radius of the perturbation is about $200 \mathrm{~km}$ when assuming a circular shape, and we think that it is located mainly above the EQ epicenter. But its western edge is not certain due to the limitation of our VLF network.

\subsection{Upper ionospheric perturbation as detected by TEC data}

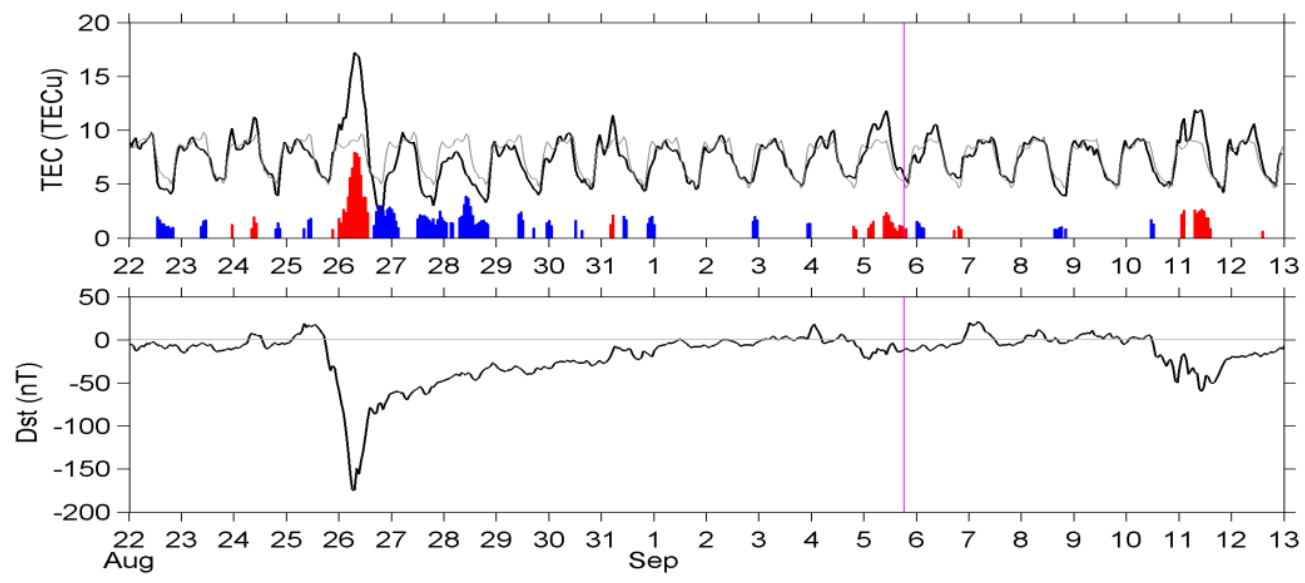

Figure. 5: The temporal evolution of TEC values in full black lines over the EQ epicenter. The thin black line is the median value during preceding 15 days, and the difference from this median (differential dTEC) is expressed above the abscissa (increase (positive) in red, and decrease (negative) in blue). A red vertical line means the EQ date. The bottom is the reproduction of Dst variation. 
We move on to the behavior of electron density perturbation in the F region of the upper ionosphere. The F region has the largest electron density and contains a widest range of altitude in the ionosphere. In the present study, we have used TEC (total electron content) representing the electron density in the upper ionosphere, because TEC is a reliable and easy means to obtain from a public dataset. The CODE (Center for Orbit Determination in Europe) GIM (global ionosphere map) is constructed from TEC data observed by GNSS (Global Navigation Satellite System) receivers around the world. The spatial resolution of this GIM is $5^{\circ}$ (in longitude) by $2.5^{\circ}$ (in latitude), and the temporal resolution is one hour. The details of TEC derivation and interpolation of the CODE GIM dataset are found in Schaer [1999]. We have checked the CODE GIM data file around the Hokkaido EQ date, and have found that two receivers at Shintotsukawa (Hokkaido) and Oshu (Iwate) are employed to build the TEC map, and so we think that the data quality around the epicenter is acceptable. Figure 5 (top panel) illustrates the variation in TEC (in full black line) reflecting the information in the upper $\mathrm{F}$ region over the EQ epicenter, again together with the variation of Dst (bottom panel) because the upper ionosphere is known to be strongly influenced by geomagnetic activity. The mean (during preceding one month) variation is given in thin lines, and the difference from this mean (differential TEC (dTEC)) is expressed above the abscissa: positive anomaly in red and negative anomaly in blue; only anomalies exceeding $1 \sigma$ are plotted here. Geomagnetic storm effects on TEC are obvious in late August and on 11 September. For the anomaly right before the EQ on 5 September, its polarity (positively anomalous) agrees with statistical results of the pre-EQ TEC anomalies over Japan [Kon et al., 2011; Liu et al., 2013]. However, we are afraid that this is also a geomagnetic effect, because this kind of positive anomaly appears almost everywhere around the world, though the Dst index is about $-20 \mathrm{nT}$, only a small disturbance. This is clearly depicted in Figure 6 of the global map on a particular date of 5 September of our greatest concern, of the accumulated hours of TEC positive anomalies (as exceeding either positive or negative $1 \sigma$ ). The accumulated hours of anomaly are 13 hours around the EQ epicenter, more than half a day, and this anomaly is more evident around Japan as compared with neighboring areas. However, 23.9\% (1240/5183) of grid points around the world reach or even exceed this value (13 hours), and also the whole day ( 24 hours) positive anomaly could be found around Western Asia. Overall, the positive anomaly around the EQ epicenter is a significant one but not a unique one. It may be a superposition of both local (e.g., seismogenic) and global (e.g., geomagnetic) effects. When comparing this anomaly with the previous VLF/LF results, the VLF anomalies tend to take 
place on 4, 5 and 6 September, while this TEC anomaly appeared only on 5 September. So we cannot say anything definite whether this TEC anomaly is due to either a geomagnetic effect or a seismic effect.

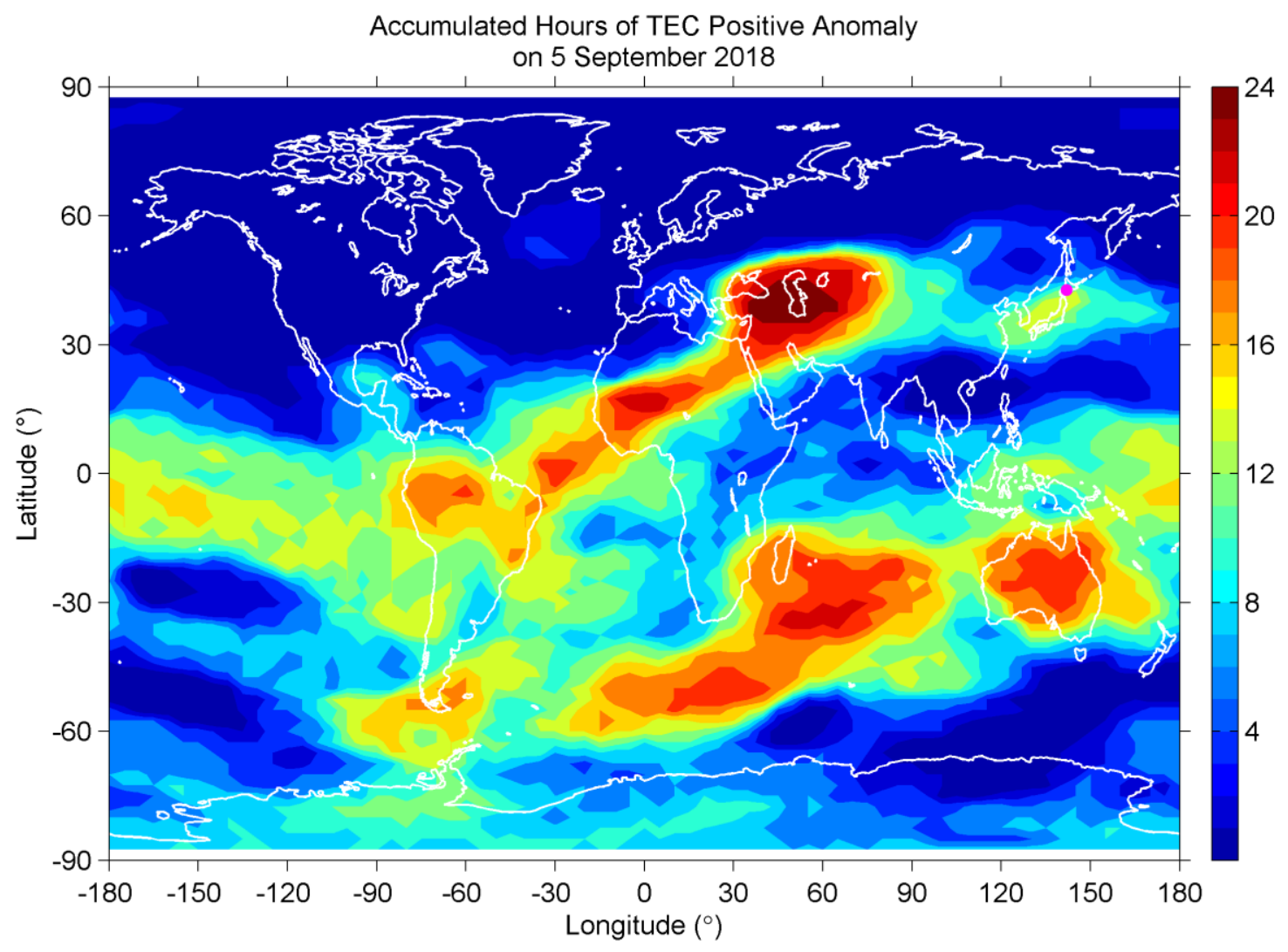

Figure 6: Global map of accumulated hours of TEC positive (in red) anomaly presumably related with an EQ on a particular day of 5 September, 2018 when a significant VLF anomaly appeared.

\subsection{Stratospheric effects. AGW effect}

Yang et al. [2019] have proposed the use of ERA5 data in order to investigate the AGW activity in the stratosphere in the height range of $15 \mathrm{~km}$ to $50 \mathrm{~km}$ in order to obtain the direct evidence on the presence of seismogenic AGWs. See the details of analysis method in Yang et al. [2019]. Figure 7 illustrates the AGW activity expressed in terms of Ep (potential energy) during a period maps from 22 August to 12 September just over the EQ epicenter. The bright band of high Ep value around $45 \mathrm{~km}$ height is correlated with temperature inversion at the stratopause, so we do not pay particular attention to it but we focus on another layer of high Ep value between 32 to $35 \mathrm{~km}$ height. The AGW activity remains high except a 
short interruption on 26-29 August, and it seems that the AGW activity was not enhanced before the EQ. Figure 8 illustrates the daily variation of spatial distribution of Ep activity in the height range of 32-35km during the period from 30 August to 6 September. AGWs are active around Japan during this period, but these waves are likely to be associated with meteorological phenomena. A cold front passed Hokkaido on 31 August, when another stationary front had remained around Japan for a long period from 24 August to 2 September. Then, a typhoon passed western Japan on 4 September, it became an extratropical cyclone, and fronts developed again over Japan on 5 and 6 September. During the possible EQ preparation period, there are many convective weather systems around Japan, and the wave activity is always high as seen in Figures 7 and 8. The meteorological effects cannot be excluded during this period, so we are not sure whether seismic effects exist or not for this EQ. However, even if it may exist, the seismic effects should be negligible and insignificant as compared with meteorological effects. As a result, we could not find any distinct seismo-related AGWs in the stratosphere as in the cases of the 2016 Kumamoto EQ [Yang et al., 2019] and the 2011 Tohoku EQ [Yang and Hayakawa, in preparation].

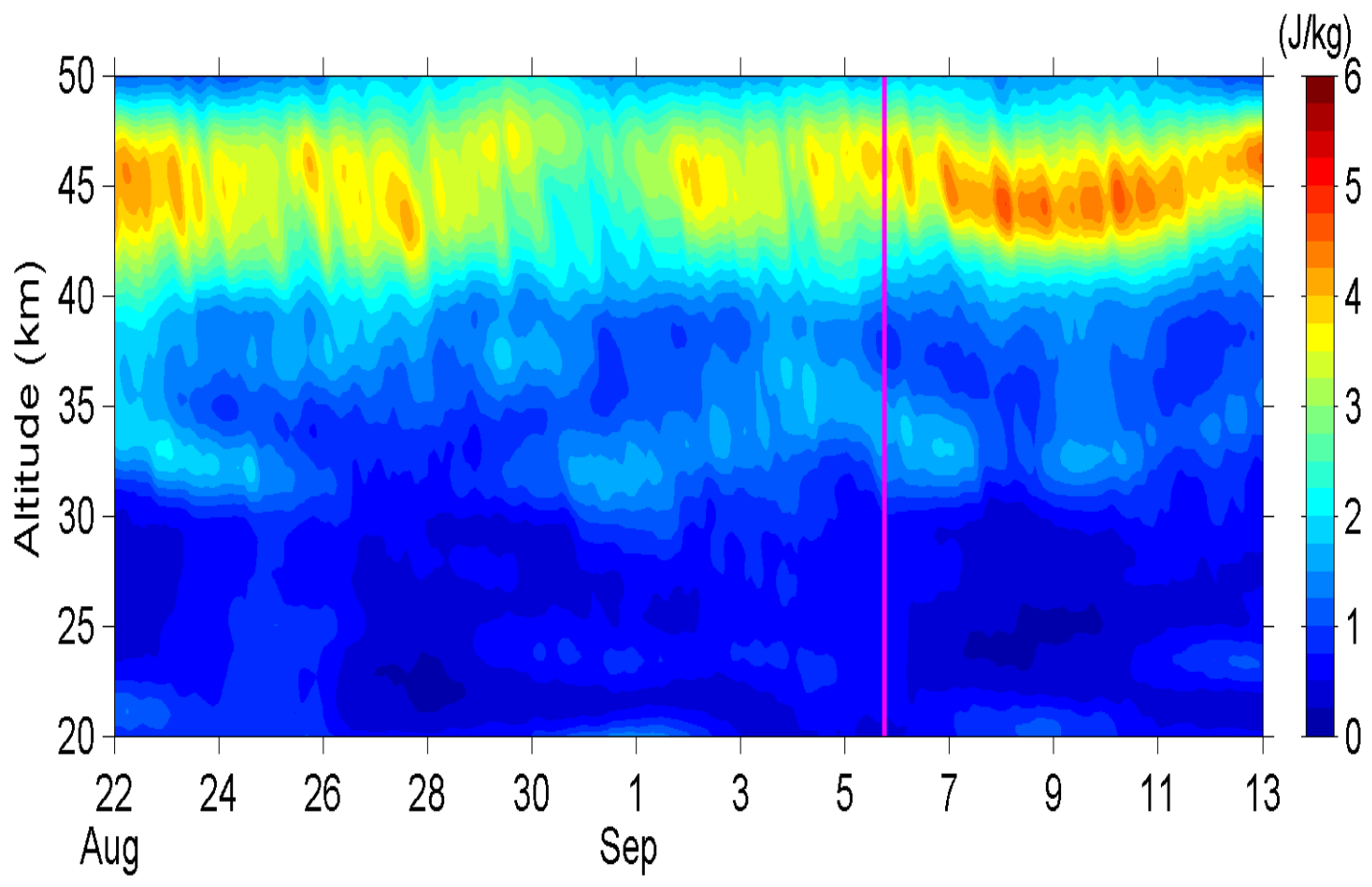

Figure. 7: Temporal evolution of Ep values in the stratosphere in the height range of 20 to $50 \mathrm{~km}$ over the EQ epicenter. The red vertical line denotes the EQ occurrence time. 
(a) 30 August, $32 \mathrm{~km}$ Altitude

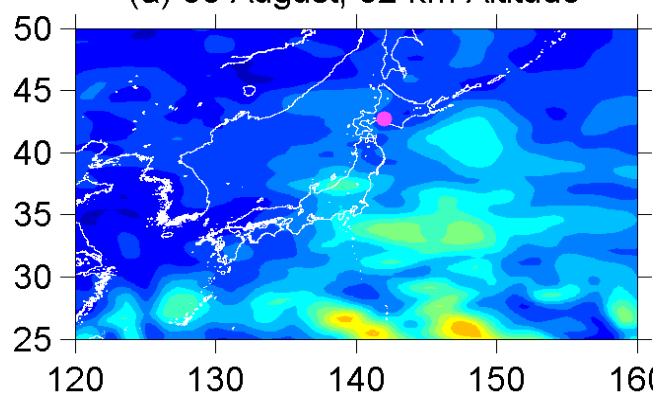

(c) 01 September, $32 \mathrm{~km}$ Altitude
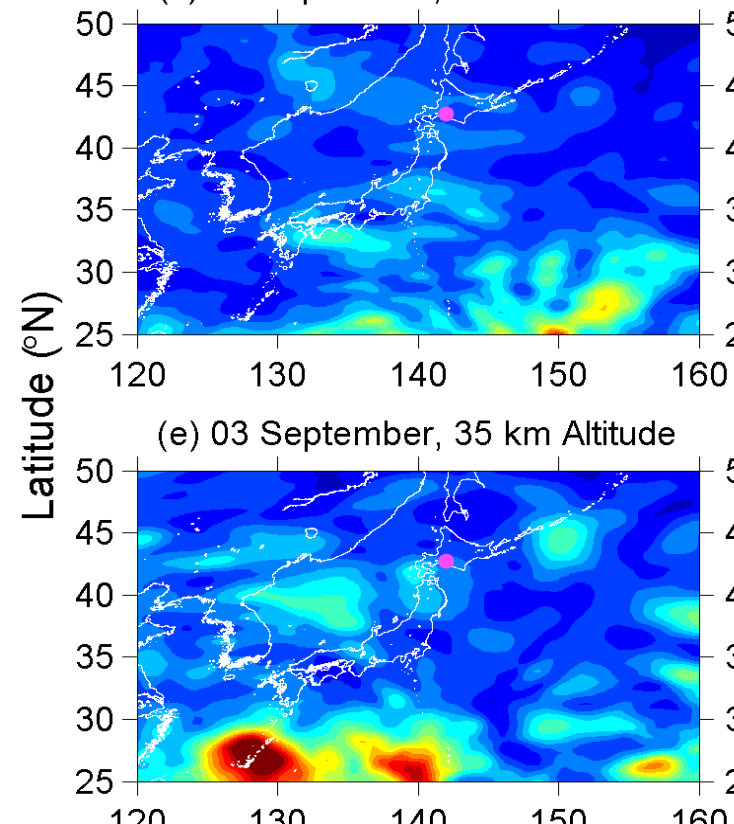

$\begin{array}{lllll}120 & 130 & 140 & 150 & 160\end{array}$

(g) 05 September, $35 \mathrm{~km}$ Altitude

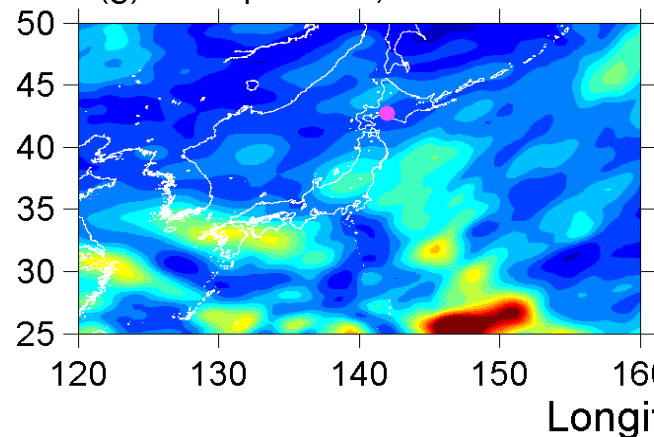

(b) 31 August, $32 \mathrm{~km}$ Altitude

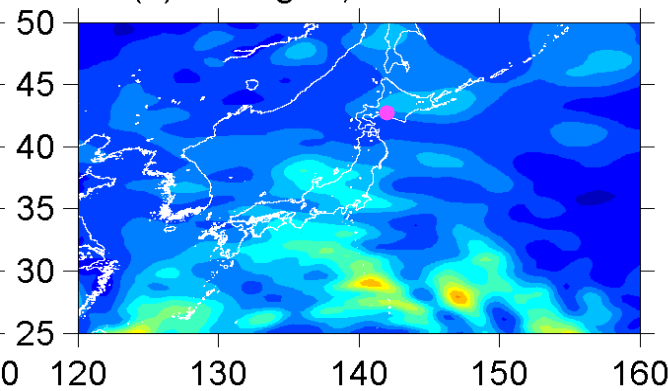

(d) 02 September, $33 \mathrm{~km}$ Altitude

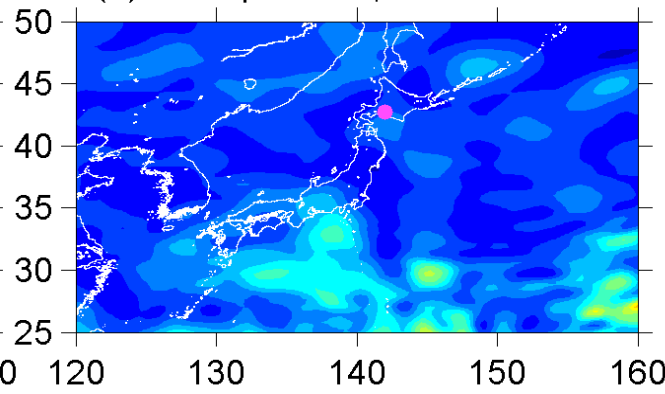

(f) 04 September, $36 \mathrm{~km}$ Altitude

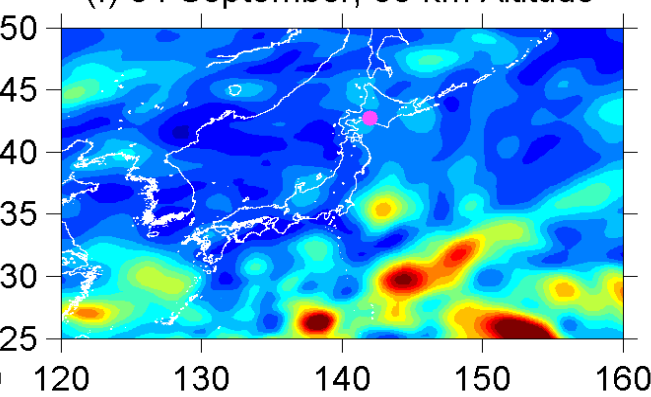

(h) 06 September, $35 \mathrm{~km}$ Altitude $\quad(\mathrm{J} / \mathrm{kg})$

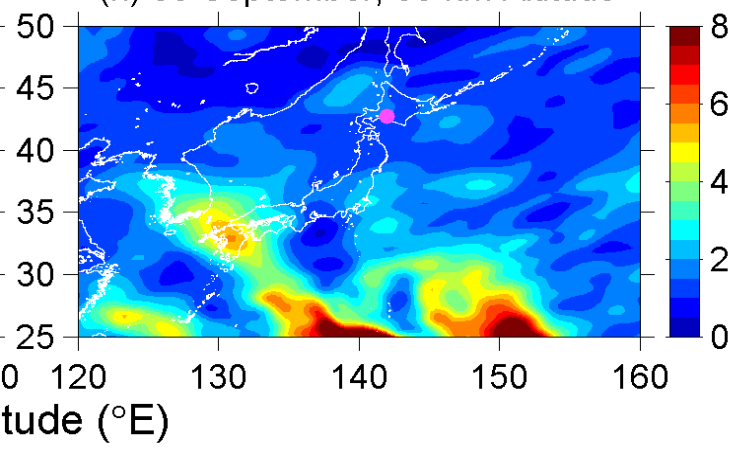

Figure. 8: Daily evolution of global distribution of Ep values at the specific altitude range of $32-35 \mathrm{~km}$ during eight days from 30 August to 06 September, 2018. A red dot in the Hokkaido Island means the EQ epicenter. 


\section{Summary and conclusion}

Based on the analyses of subionospheric VLF data from Japan and Russia, GIM TEC data, and ERA5 temperature profile data, the following observational facts have emerged for a rather huge Hokkaido EQ on 5 September, $2018(\mathrm{M}=6.7)$.

(1) The most distinct anomaly has been identified from VLF data observed both in Japan and Russia. The clear precursory propagation anomalies have been observed on 4 September in Japan from the propagation paths of JJY-WKN and JJI-WKN and on 4-6 September from the JJY-PTK path. It is evident that the lower ionosphere above the EQ epicenter is disturbed before the EQ, and our network observation has yielded that the ionospheric perturbation is located mainly above the EQ epicenter with radius about $200 \mathrm{~km}$.

(2) As for the GIM TEC data in the upper F region above the EQ epicenter, even though we have found an anomaly on 5 September, it is very uncertain whether it is attributable either to the geomagnetic storm effect or to the seismic effect.

(3) The stratospheric AGW activity has been investigated using the ERA5 temperature profile data and we have found that there were many AGW anomalies, but all are highly likely due to strong meteorological effects. So the seismic effect is not so clear.

With taking into account the above observational findings for this particular EQ, the most obvious is a clear precursor in the lower ionosphere before the EQ, and the region of this seismo-ionospheric perturbation is likely to be located mainly above the EQ epicenter with radius of about $200 \mathrm{~km}$. Despite the presence of overhead location of the seismo-ionospheric perturbation, our study on whether any AGW activity is present in the stratosphere, has yielded a negative result: the absence of AGW activity in the stratosphere. These experimental data seem to provide us intuitively with negative evidence to the Atmospheric oscillation hypothesis in the LAIC, in sharp contrast to our recent papers for the 2016 Kumamoto EQ [Yang et al., 2019] and the 2011 Tohoku EQ [Yang et al., 2019], in which we have detected directly remarkable AGW activities in the stratosphere and the corresponding lower ionospheric perturbations consistent (both in time and in spatial distribution) with the stratospheric AGWs. One more important point we have to notice, is the significant spatial shift of the location of seismo-ionospheric disturbance with respect to the EQ epicenter [Yang et al., 2019]. 

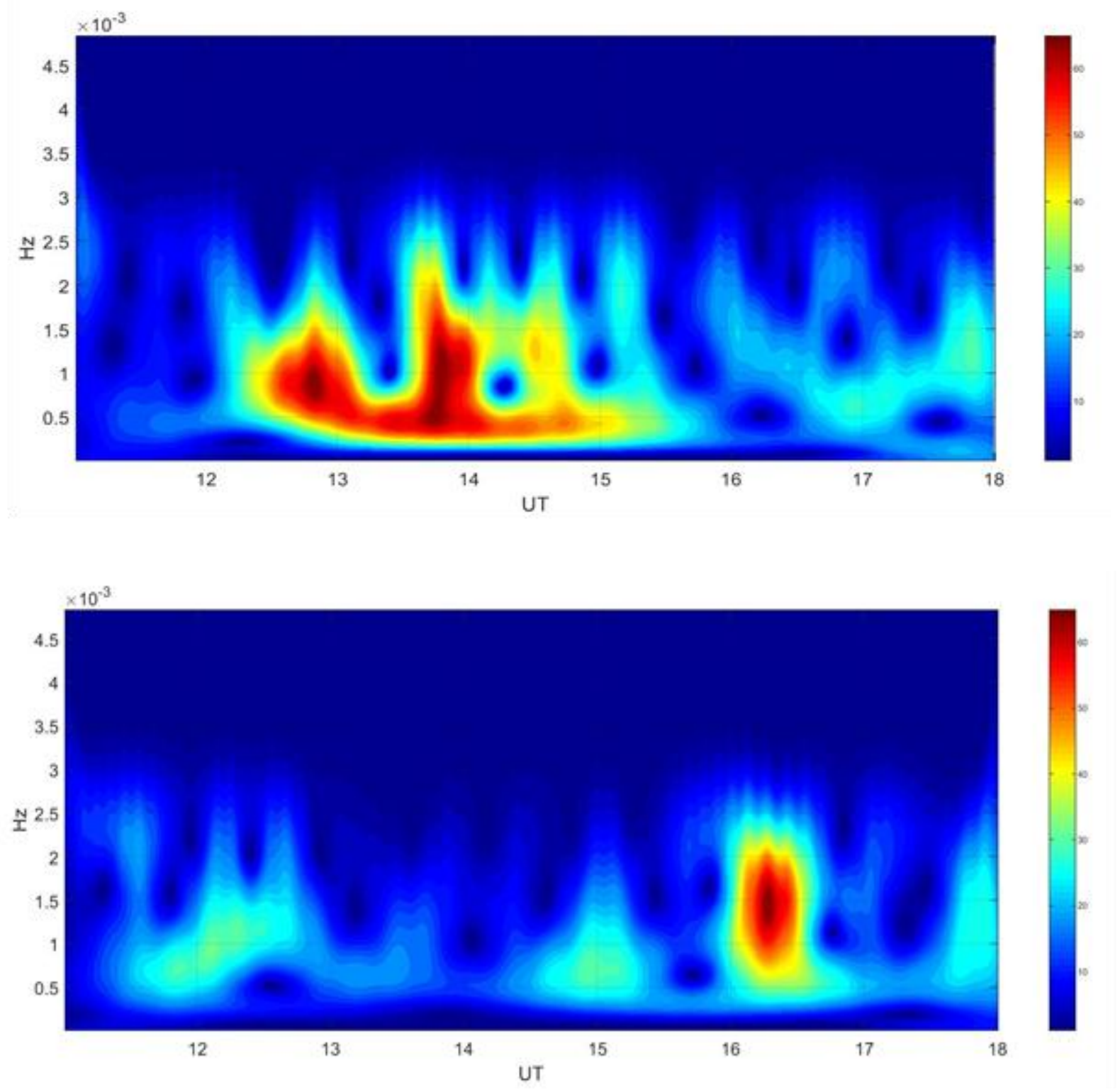

Figure 9: Wavelet analysis results for nighttime (UT=11-18h) LF data on the propagation paths of JJY-YHS on two relevant days of 4 (upper panel) and 5 (lower panel) September, respectively. The power spectral density is indicated in terms of the color on the right. The ordinate indicates wave frequency eg, $\mathrm{f}=0.5 \times 10^{-3} \mathrm{~Hz}(\mathrm{~T}$ (period) $=33$ minutes), $1 \times 10^{-3} \mathrm{~Hz}(\mathrm{~T}=16.7 \mathrm{~m}), 1.5 \times 10^{-3} \mathrm{~Hz}(\mathrm{~T}=11 \mathrm{~m}), 2 \times 10^{-3} \mathrm{~Hz}(\mathrm{~T}=8.3 \mathrm{~m}), 2.5 \times 10^{-3} \mathrm{~Hz}(\mathrm{~T}=6.6 \mathrm{~m}), 3 \times 10^{-3} \mathrm{~Hz}$ $(\mathrm{T}=5 \mathrm{~m})$ etc).

In order to examine this seeming inconsistency with the Atmospheric oscillation channel, we have thought that higher-frequency components (AW with periods less than 10 minutes) even in the Atmospheric oscillation channel might be involved in the LAIC effect, because these AW components tend to propagate vertically upwards unlike the AGWs, leading to the generation of ionospheric 
perturbation mainly above the EQ epicenter. Additionally we have to add that it is rather difficult to extract these AW components in the ERA5 data analysis. So we have checked the fluctuation spectra in the VLF amplitude data on the propagation path of JJY-YHS on the two days of 4 and 5 September before the EQ, and Figure 9 is the corresponding wavelet analysis results of VLF fluctuation. The upper panel of Figure 9 refers to 4 September, while the lower, to 5 September. It is clear from these figures that main frequency (period) components in VLF fluctuations are found to lie in the AGW range of T=20-30 minutes, but extending down to AW range. This result is inconsistent with our expectation.

The most salient feature of our Atmospheric oscillation hypothesis in the LAIC is apparent lateral (spatial) shift of ionospheric perturbation from the EQ epicenter as already emphasized in the cases of the 2016 Kumamoto EQ [Yang et al., 2019] and the 2011 Tohoku EQ. The present work suggests that the seismo-ionospheric perturbation seems to be generated over the EQ epicenter without any significant spatial shift. So that, it is unlikely that the atmospheric oscillation hypothesis is not working for this Hokkaido EQ, and any other mechanism like Chemical or Electrostatic channel (both through electric fields) might be in operation. In this case we have to think about how to interpret the presence of AGW modulation in the VLF data. Needless to say, the primary agent of LAIC is definitely situated in the lithosphere, and we have to speculate the origin of those AGW components. There have been proposed a few hypothetical possibilities on this AGW modulation in the lithosphere: (1) source modulation by the global seismo-gravitational pulsations of the Earth (with periods of 1-3 hours) [Gokhberg et al., 1994], (2) excitation of wide spectra of buoyancy oscillations as a result of destabilization of the thin near-surface atmospheric layer above the thermal anomaly [Tronin, 2002]. A further recent and interesting suggestion has been done by Chen et al. [2018], who have shown, based on the GPS crustal movement observations, that the typical ground vibration in the period range from 1.5 to 3.5 hours (AGW range) were commonly observed 10- 0 days before the major EQs (as a short-term precursor), and that they have attributed these ground vibrations to the crustal resonance at the natural frequency in the AGW range due to preEQrelated stress loading in the crust. If we base on this, the presence of crustal resonance at AGW frequencies results in the uplift and depression of Earth's surface in wide areas that would be the dominant ground motion exciting numerous pre-EQ anomalies including the atmospheric and ionospheric perturbation. So we can expect the existence of AGW modulation even in VLF data because any kinds of parameters in the lithosphere or lower atmosphere are modulated by the frequency of AGWs for any other LAIC hypotheses, either Chemical or Electrostatic. 
An alternative explanation of the inconsistency even by supporting the Atmospheric oscillation hypothesis may be the intrinsic propagation characteristics of AGWs from a transient source [Alexander and Holton J, 2004]. Taking into account the conclusions of this paper, we might suggest that seismogenic atmospheric oscillations once excited near the ground, have a smaller period (in the AW range) around the EQ epicenter and in the lower atmosphere, but those waves have a longer period (in the AGW range) away from the wave source and in the upper atmosphere and ionosphere, leading to the VLF fluctuations in the range of 20-30 minutes for our Hokkaido EQ as in Figure 9.

Finally it is uncertain at the moment which one of the above two tentative explanations of the abovementioned discrepancy is more plausible, the authors may feel that the first possibility based on the crustal resonance in the lithosphere might be more probable, but unfortunately we have no lithospheric information such as ULF electromagnetic emissions [Hayakawa and Molchanov, 2002; Molchanov and Hayakawa, 2008; Hayakawa, 2015] because there is no geomagnetic observatory close to the EQ epicenter. As for this Hokkaido EQ, we need to try to find any other possible data related to the atmospheric and lithospheric activity to elaborate the present work and to give an answer to the above problem.

As the most general problem, we have to emphasize here that the most essential point of the study of LAIC effect will be the detailed comparison (both the temporal evolution and spatial distribution) of EQrelated ionospheric perturbation with the corresponding atmospheric, stratospheric and lithospheric data. Especially spatial distributions of different parameters which have been poorly investigated so far, would play the main role in the LAIC studies. We are generally in favor of the Atmospheric oscillation hypothesis in the LAIC, so that we want to increase the number of EQ events in which the Atmospheric oscillation hypothesis is working as the main player. However, we do not deny other channels because it seems possible that Chemical, Electrostatic, or Atmospheric oscillation channel is switched on by different processes in the lithosphere and act independently [Lizunov and Hayakawa, 2004].

\section{Acknowledgement}

The GIM TEC data are provided by CODE (Center for Orbit Determination in Europe) at University of Bern, Switzerland. The ERA5 data is processed and carried out by ECMWF within the Copernicus Climate Change Service (C3S). 


\section{Reference}

1. Alexander, M. J., and Holton, J. R.; On the spectrum of vertically propagating gravity waves generated by a transient heat source, Atmospheric Chemistry and Physics, vol. 4, 923-932, 2004.

2. Chen, C. H., Lin, L. C., Yeh, T. K., et al. Crustal resonance before moderate-to-large earthquakes, submitted to Nature Geoscience, 2018.

3. Dobrovsky, I. I., Zukov, S. I., and Myachkin, V. I.; Estimation of the size of earthquake preparation zone, Pure Appl. Geophys., vol. 117, 1025-1044, 1979.

4. Freund, F.; Stress-activated positive hole carriers in rocks and the generation of pre-earthquake signals, in "Electromagnetic Phenomena Associated with Earthquakes", Ed. by M. Hayakawa, Transworld Research Network, Trivandrum, 41-96, 2009.

5. Gokhberg, M. B., Nekrasov, A. K., and Shalimov, S. L; A new approach to the problem of lithosphere-ionosphere coupling before the earthquake, in "Electromagnetic Phenomena Associated with Earthquakes", Ed. by M. Hayakawa and Y. Fujinawa, TERRAPUB, Tokyo, 619626, 1994.

6. Hayakawa, M.; Earthquake precursor studies in Japan, in "Pre-Earthquake Processes: A Multidisciplinary Approach to Earthquake Prediction Studies”, Ed. by D. Ouzounov et al., AGU, Wiley, 7-18, 2018.

7. Hayakawa, M., and Molchanov, O. A.; Seismo-Electromagnetics: Lithosphere-AtmosphereIonosphere Coupling, TERRAPUB, Tokyo, 477p, 2002.

8. Hayakawa, M., Asano, T., Rozhnoi, A., and Solovieva, M; Very-low- and low-frequency sounding of ionospheric perturbations and possible association with earthquakes, in "PreEarthquake Processes: A Multidisciplinary Approach to Earthquake Prediction Studies", Ed. by D. Ouzounov et al., AGU, Wiley, 277-304, 2018.

9. Hayakawa, M., Kasahara, Y., Nakamura, T., Hobara, Y., Rozhnoi, A., Soloviava, M., Molchanov, O. A., and Korepanov, V.; Atmospheric gravity waves as a possible candidate for seismoionospheric perturbations, J. Atmos. Electr., vol. 31, 129-140, 2011.

10. Hayakawa, M.; Earthquake Prediction with Radio Techniques, John Wiley \& Sons, Singapore, $294 p, 2015$.

11. Hayakawa, M.; Probing the lower ionospheric perturbations associated with earthquakes by means of subionospheric VLF/LF propagation, Earthq. Sci., vol. 24, 523-534, 2011. 
12. Kon, S., Nishihashi, M., and Hattori, K; Ionospheric anomalies possibly associated with $M \geq 6.0$ earthquakes in the Japan area during 1998-2010: Case studies and statistical study, J. Asian Earth Sci., vol. 41(4), 410-420, 2011.

13. Liu, J. Y., Chen, C. H., and Tsai, H. F.; A statistical study on seismo-ionospheric precursors of the total electron content associated with $146 \mathrm{M} \geq 6.0$ earthquakes in Japan during 1998-2011, in "Earthquake Prediction Studies: Seismo Electromagnetics", Ed. by M. Hayakawa, TERRAPUB, Tokyo, 1-13, 2013.

14. Liu, J. Y., Hattori, K., and Chen, Y. I.; Application of total electron content derived from the Global Navigation Satellite System for detecting earthquake precursors, in "Pre-Earthquake Processes: A Multidisciplinary Approach to Earthquake Prediction Studies", Ed. by D. Ouzounov et al., AGU, Wiley, 305-317, 2018.

15. Lizunov, G., and Hayakawa, M.; Atmospheric gravity waves and their role in the lithospheretroposphere-ionosphere interaction, IEEJ (Institute of Electrical Engineers of Japan), Trans. Fundamentals and Materials, vol. 124, 1109-1120, 2004.

16. Molchanov, O. A., and Hayakawa, M.; Seismo Electromagnetics and Related Phenomena: History and latest results, TERRAPUB, Tokyo, 189p, 2008.

17. Ouzounov, D., Pulinets, S., Hattori, K., and Taylor, P. (Eds.); Pre-Earthquake Processes: A Multidisciplinary Approach to Earthquake Prediction Studies, Geophysical Monograph Series 234, AGU, Wiley, 365p, 2018.

18. Pulinets, S. A., and Boyarchuk, K.; Ionospheric Precursors of Earthquakes, Springer, Berlin, 315p, 2004.

19. Pulinets, S., Ouzounov, D., Karelin, A., and Davidenko, D.; Lithosphere-atmosphere-ionospheremagnetosphere coupling - A concept for pre-earthquake signals generation, in "Pre-Earthquake Processes: A Multidisciplinary Approach to Earthquake Prediction Studies", Ed. by D. Ouzounov et al., AGU, Wiley, 79-98, 2018.

20. Rozhnoi, A., Solovieva, M. S., Molchanov, O. A., and Hayakawa, M. Middle latitude LF (40 kHz) phase variations associated with earthquakes for quiet and disturbed geomagnetic conditions, Phys. Chem. Earth, vol. 29, 599-605, 2004.

21. Rozhnoi, A., Solovieva, M., and Hayakawa, M.; VLF/LF signals method for searching of electromagnetic precursors, in "Earthquake Prediction Studies: Seismo Electromagnetics", Ed. by M. Hayakawa, TERRAPUB, Tokyo, 31-48, 2013.

22. Schaer, S., Mapping and predicting the Earth's ionosphere using the global positioning system, Ph.D. dissertation, Astron. Inst. Univ. of Bern, Switzerland, 1999. 
23. Sorokin, V., Chmyrev, V., and Hayakawa, M.; Electrodynamic Coupling of LithosphereAtmosphere-Ionosphere of the Earth, NOVA Science Pub. Inc., New York, 326p, 2015.

24. Tronin, A. A.; Atmosphere-ionosphere coupling. Thermal anomalies on the Earth's surface in seismic processes, in "Seismo Electromagnetics: Lithosphere-Atmosphere-Ionosphere Coupling", Ed. by M. Hayakawa and O. A. Molchanov, TERRAPUB, Tokyo, 173-176, 2002.

25. Yang, S. S., Asano, T., and Hayakawa, M; Abnormal gravity wave activity in the stratosphere prior to the 2016 Kumamoto earthquakes, J. Geophys. Res., Space Phys., vol. 124, 2019. https://doi.org/10.1029/2019/2018JA026002 